\title{
Patrones de diversidad y aspectos etnobotánicos de las plantas arvenses del valle de Tehuacán-Cuicatlán: el caso de San Rafael, municipio de Coxcatlán, Puebla
}

\author{
Diversity and ethnobotanical facts of the weeds from Tehuacan-Cuicatlán Valley: San Rafael, \\ Coxcatlán Municipality, Puebla
}

\author{
César Albino-García, Héctor Cervantes, Martín López, Leticia Ríos-Casanova y Rafael Lira* \\ Unidad de Biología, Tecnología y Prototipos (UBIPRO), Facultad de Estudios Superiores Iztacala, Universidad Nacional Autónoma de México. Apar- \\ tado postal 314, Avenida de los Barrios 1, Los Reyes Iztacala, 54090, Tlalnepantla Estado de México, México. \\ *Correspondencia: rlira@servidor.unam.mx
}

\begin{abstract}
Resumen. Este trabajo documenta la riqueza, composición florística, diversidad y conocimiento tradicional de las plantas arvenses de las milpas de San Rafael, Municipio de Coxcatlán, Puebla. De acuerdo con los antecedentes, se probaron 4 hipótesis: 1), la riqueza y diversidad de las plantas arvenses de San Rafael son similares a las registradas en estudios comparables realizados dentro del valle de Tehuacán; 2), más del 50\% de estas plantas se usan, y principalmente como forraje; 3), con algunas arvenses, se practica 1 o más de los tipos de manejo tradicional (p. ej., toleradas, protegidas, o fomentadas o inducidas) y 4), los agricultores de mayor edad son quienes poseen más conocimientos acerca de estas plantas. Se hicieron muestreos en 12 milpas mediante líneas de Canfield y se entrevistó a 20 agricultores de 32 a 80 años de edad. Se encontraron 42 especies de 12 familias de plantas vasculares, un valor de riqueza intermedio con respecto a los registrados (5-90 spp.) en estudios previos realizados en otras zonas del valle de Tehuacán-Cuicatlán. La riqueza registrada en las milpas fue de 6 a 17 especies $(\overline{\mathrm{x}}=10.3 \sigma=3.63)$ y los valores de diversidad de Shannon variaron entre 0.795 y $2.209(\bar{x}=1.494 \sigma=0.4440)$, lo cual está dentro de los límites esperados para comunidades de plantas arvenses. Los agricultores reconocieron entre 20 y 31 especies, pero no se encontró correlación significativa entre su edad y el número de plantas identificadas. Sólo 11 especies se reconocieron como útiles, pero para ninguna hay evidencia de que esté sujeta a algún tipo de manejo. De acuerdo con estos resultados, sólo las hipótesis 1 y 2 pudieron corroborarse, lo que sugiere que aún no es posible establecer generalizaciones en relación con los temas aquí estudiados para las plantas arvenses.
\end{abstract}

Palabras clave: arvenses, composición florística, diversidad, plantas útiles, valle de Tehuacán-Cuicatlán

\begin{abstract}
This study documents floristic composition, richness, diversity and traditional knowledge of the weeds inhabiting milpas (cornfields) of San Rafael, Municipality of Coxcatlán, Puebla. According to previous studies, 4 hypotheses were tested: 1), species richness and diversity of the weeds of San Rafael are similar to those recorded in other cornfields in several sites of the Tehuacán-Cuicatlán Valley; 2), more than $50 \%$ of the weeds of San Rafael are used and mainly as fodder; 3), some of the weeds are being submitted to 1 or more of the traditional management practices (v.gr. tolerance, protection, or enhancement) and 4), older farmers possess more knowledge about the weeds. Twelve cornfields were sampled using Canfield's lines, and 20 farmers aged 32-80 were interviewed. Forty two species of 12 families of vascular plants were found. This is an intermediate value with respect to the species richness (5-90 spp.) recorded for other sites in the Tehuacán Valley. Average species richness of weeds in the cornfields studied was between 6 and $17(\overline{\mathrm{x}}=10.3 \sigma=3.63)$, whereas the values of Shannon Index were between 0.795 and $2.209(\overline{\mathrm{x}}=1.494 \sigma=$ 0.4440 ), which is within the limits expected for weed communities. Farmers recognized between 20 and 31 species and there were not a significant correlation between farmer age and the number of plants they identified. Only 11 species were recognized as useful, and there were not evidences of handling for any of them. According to these results, only the hypotheses 1 and 2 could be corroborated, which suggests it is not yet possible to establish generalizations for the topics analyzed for weeds in this study.
\end{abstract}

Key words: weeds, diversity, floristic composition, useful plants, Tehuacán-Cuicatlán Valley. 


\section{Introducción}

Las plantas arvenses generalmente se consideran "malezas" que compiten con los cultivos y reducen su productividad; por ello, y con el fin primordial de erradicarlas, han sido ampliamente estudiadas bajo diversos enfoques (evolutivo, ecológico, agronómico, sobre su resistencia a herbicidas, etc. (Baker, 1965; Harlan y De Wet, 1965; Cardina et al., 1997; Dekker, 1997; Liebman et al., 2001; Booth et al., 2003). En contraste, en el contexto de la agricultura tradicional de México, numerosas especies de plantas arvenses se reconocen como recursos importantes para satisfacer diversas necesidades humanas, principalmente de alimento, medicina y forraje (Bye, 1979, 1981, 1993; Chacón y Gliessman, 1981; Davis y Bye, 1982; ZizumboVillarreal y Colunga-García Marín, 1982; Alcorn, 1984; Williams, 1985; Mapes et al., 1997; Liebman et al., 2001; Paredes-Flores, 2001，2006; Blanckaert, 2001，2007; Blanckaert et al., 2004, 2007; Paredes-Flores et al., 2007). En algunos casos, incluso, también se ha documentado su valor económico actual y potencial (Bye y Linares, 1983; Vieyra-Odilón y Vibrans, 2001).

Para algunas especies de arvenses comestibles o medicinales, diversos estudios han registrado prácticas de manejo tradicional dirigidas a su preservación o incluso a incrementar su abundancia (Bye, 1979, 1981, 1993; Davis y Bye, 1983; Williams, 1985; Vázquez-Rojas, 1991; Mapes et al., 1997; Lira y Casas, 1998; Casas et al., 2001, 2006, 2007; Blanckaert, 2001; Blanckaert et al., 2004, 2007; Paredes-Flores, 2006; Paredes-Flores, et al., 2007, entre otros). Estas prácticas, que se han documentado tanto para especies silvestres en áreas forestales como para arvenses en campos de cultivo, han sido categorizadas y definidas por Casas (2007) como: 1) tolerancia, cuando las especies de plantas útiles o fenotipos particulares de ellas se dejan en su sitio durante los desmontes de la vegetación natural o deshierbes en los campos agrícolas; 2) fomento o inducción, que comprende actividades dirigidas a incrementar la densidad poblacional y la disponibilidad de las plantas útiles mediante aclareos selectivos de la vegetación, favoreciendo algunos elementos y erradicando otros, empleando riego artificial en áreas silvestres, o dispersando propágulos sexuales o vegetativos de especies o fenotipos de interés; 3) protección, la cual se lleva a cabo favoreciendo la permanencia de algunas plantas manteniéndolas bajo cuidados especiales, que pueden incluir la reducción de competencia y de herbívoros, podas, protección contra plagas, heladas, radiación solar y sombra, y 4) cultivo $y$ trasplante, que incluye la siembra de semillas o plantación de propágulos vegetativos o incluso el trasplante de individuos completos a áreas manejadas, tales como sistemas agroforestales y huertos. Tal diversidad de formas de manejo para las especies arvenses, sugiere que existe una intensa pero aún poco documentada relación entre la gente $\mathrm{y}$ estas plantas.

Considerando que la flora arvense de México se ha estimado entre 2298 y 2814 especies (Villaseñor y Espinosa-García, 1998; Espinosa-García et al., 2004), es de esperarse que en muchas partes del país las relaciones de la gente con este tipo de plantas sean muy frecuentes y diversas. Una de las regiones de México donde las interacciones de la gente con las plantas de su entorno son particularmente intensas es el valle de Tehuacán-Cuicatlán. Para esta región se han registrado más de 1600 especies de plantas útiles, de las cuales alrededor del $8 \%$ son plantas arvenses que están sujetas a algún tipo de manejo tradicional como los descritos anteriormente (Casas et al., 2001; Lira et al., 2009).

Este trabajo aspira a ser una contribución que amplíe el panorama sobre las comunidades de plantas arvenses, sus usos y manejo, mediante un estudio de caso llevado a cabo en San Rafael, municipio de Coxcatlán, Puebla. Para esta localidad, investigaciones previas han registrado información sobre 360 especies de plantas útiles (p. ej. Blanckaert, 2001; Rosas-López, 2003; Blanckaert et al., 2004; Canales et al., 2005, 2006; Casas et al., 2006; Rodríguez- Arévalo et al., 2006; Lira et al., 2008, 2009; Avendaño et al., 2009). De estas investigaciones, la de Rosas-López (2003) estudia con mayor profundidad las plantas arvenses; en este estudio etnobotánico, la autora registra 59 especies de plantas útiles, en ambientes que denominó "áreas antropogénicas", en las que incluye terrenos de cultivo, potreros y caminos rurales. La mayor parte de estas especies (38) se registraron como forrajeras y 9 como manejadas ( 6 toleradas, 2 protegidas y una trasplantada).

Más recientemente, Paredes-Flores (2007) registró las plantas arvenses de Zapotitlán, Salinas; su objetivo fue documentar el uso y manejo de una de estas plantas (Proboscidea louisianica (Mill.) Thell. ssp. fragans (Lindl.) Bretting). En ese trabajo, mediante colectas en 20 milpas activas y 10 inactivas, se registraron 85 especies de plantas arvenses, de las cuales 55 son plantas útiles, pero sólo se informa sobre las prácticas de manejo para $P$. louisianica.

Otros trabajos enfocados a estudiar aspectos etnobotánicos y ecológicos de los recursos vegetales de diferentes sitios del valle de Tehuacán utilizaron cuadrantes como método de muestreo y documentaron entre 5 y 90 especies de plantas arvenses, de las cuales se utiliza entre el $40 \mathrm{y}$ $100 \%$ (Cuadro 1). En trabajos más directamente enfocados al estudio de la riqueza y diversidad de la flora arvense en sistemas de cultivo (melón, papaya, limón y milpa) en Santa María Tecomavaca, Oaxaca (Blanckaert, 2007; Blanckaert et al., 2007), el método que se usó para los muestreos fue la Línea de Canfield (Canfield, 1941); se 


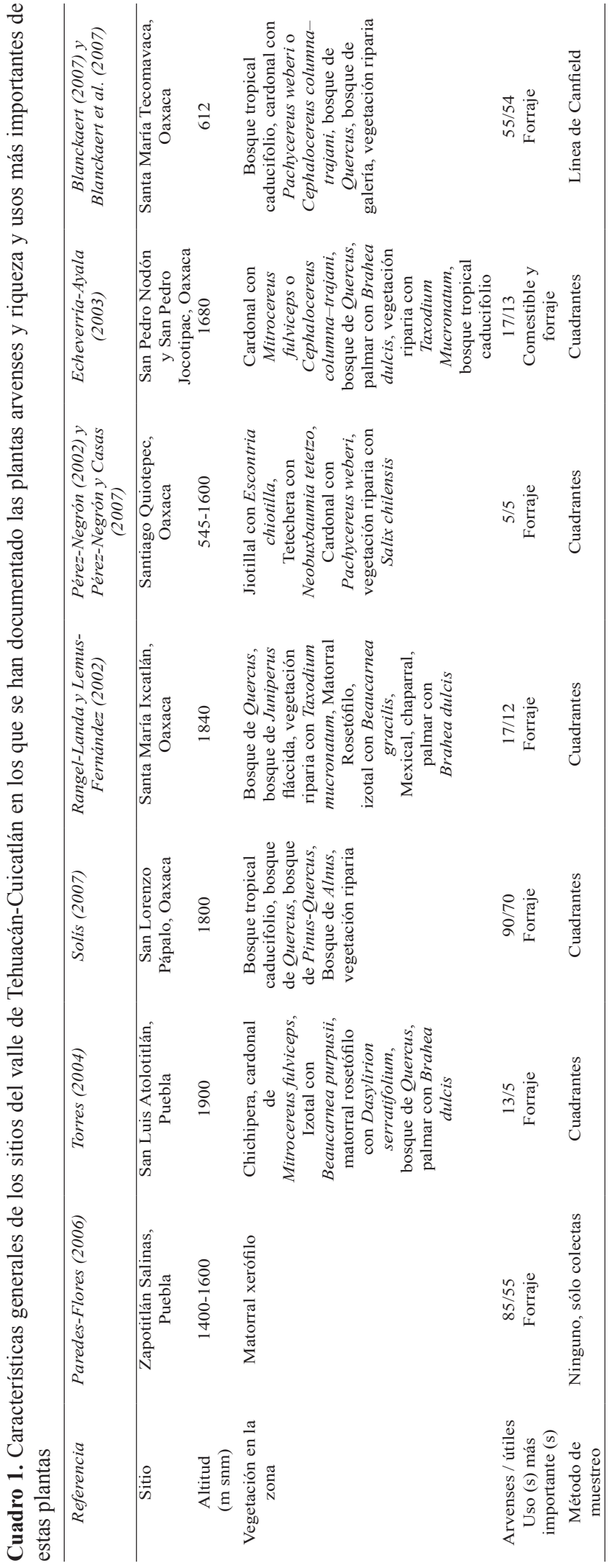

registraron 161 especies de plantas arvenses, de las cuales 55 fueron ubicadas en el sistema de milpa. Para 54 de estas especies se registraron uno o más usos, aunque sólo para 7 (Amaranthus hibrydus, Ipomea purporea, Mentzelia apera, Porophyllum ruderale, Tithonia tubiformis, Viguiera dentata y Waltheriana americana) se pudo documentar alguna forma particular de manejo tradicional, como la tolerancia y el fomento o inducción. Ninguna de las especies registradas como útiles fue más abundante ni presentó valores de cobertura significativamente mayores que las no útiles, lo que sugiere que las prácticas de manejo tradicional antes mencionadas no han sido del todo exitosas.

En el Cuadro 1 se muestra una síntesis comparativa de las características generales de los sitios donde se realizaron todos estos trabajos y de su riqueza de plantas arvenses. Un primer aspecto etnobotánico que puede reconocerse como recurrente en todos estos trabajos, es que la mayoría de las plantas arvenses se utilizan y que el uso más importante es el forraje. Adicionalmente, en los estudios de Paredes-Flores (2006), Blanckaert (2007) y Blanckaert et al. (2007), se registró que las personas de mayor edad son quienes poseen más conocimientos acerca de las plantas arvenses, lo cual también se ha documentado en numerosos estudios etnobotánicos realizados en varias partes del mundo (Ladio y Lozada, 2001, 2003; Fassil, 2003; Blanckaert et al., 2004; Canales et al., 2005, 2006; Carretero, 2005; Hernández et al., 2005; Kamalakannan y Balakrishnan, 2009, entre otros).

Con la intención de ampliar la información sobre las plantas arvenses del valle de Tehuacán-Cuicatlán, este trabajo tuvo como objetivo documentar la composición florística, distribución, diversidad y conocimiento tradicional (nombres comunes, usos, relación edad-conocimiento y manejo) de las plantas arvenses asociadas al sistema agrícola de milpa de la comunidad de San Rafael. En particular se buscó contestar las siguientes preguntas:

1. ¿Cuál es la riqueza y diversidad de la flora arvense de las milpas de San Rafael y qué tan comparable es con la registrada en otros sitios del valle de Tehuacán-Cuicatlán? 2. ¿Se usan especies arvenses en San Rafael? 3. ¿Se les da algún tipo de manejo a las especies arvenses en San Rafael?, ¿el manejo influye en su abundancia y riqueza? 4. ¿El conocimiento tradicional sobre usos y manejo tiene relación con la edad de los agricultores?

Con base en la información etnobotánica y ecológica documentada para la región, así como en observaciones de campo, en este estudio se analizaron las siguientes hipótesis para las preguntas planteadas:

1. La riqueza y diversidad de las plantas arvenses de las milpas de San Rafael serán similares (entre 5 y 90 spp. 
y valores de diversidad menores a 2.0) a las registradas en estudios comparables realizados dentro del valle de Tehuacán. 2. Se espera que una proporción relativamente alta $(>50 \%)$ de las especies arvenses de las milpas de San Rafael se usen con diferentes propósitos, pero principalmente como plantas forrajeras. 3. Se espera que el manejo que se da a algunas de las plantas arvenses útiles de San Rafael sea uno o más de los de tipo tradicional (p.ej. la tolerancia, la protección o el fomento o inducción), con lo cual se intente asegurar su preservación o incluso incrementar su abundancia. 4. Los agricultores de mayor edad serán quienes posean más conocimientos acerca de las plantas arvenses útiles de San Rafael.

\section{Materiales y métodos}

Ubicación y características de la zona de estudio. La comunidad de San Rafael se ubica en la porción suroeste del valle de Tehuacán-Cuicatlán (Fig. 1), a una altitud de $1217 \mathrm{~m} \mathrm{snm}$. Su clima es semiárido con un promedio anual de temperatura de $22^{\circ} \mathrm{C}\left(25^{\circ} \mathrm{C}\right.$ en abril y mayo, y $18^{\circ} \mathrm{C}$ en enero); la época de lluvias se presenta entre junio y septiembre y el área recibe una precipitación promedio anual de $394.6 \mathrm{~mm}$. Los suelos son regosol fútrico y xerosol háplico, los cuales soportan vegetación de bosque tropical caducifolio y matorral xerófilo, con especies como Bursera morelensis, B. aptera, Pachycereus weberi, Opuntia puberula, Ceiba aesculifola ssp. parvifolia, Acacia cochliacantha, Escontia chiotilla, Neobuxbaumia tetetzo, Cephlocereus columna-trajani y Agave macroacantha, entre otras (Medina-Sánchez, 2000). La población de San Rafael es de poco más de 300 personas, quienes habitan en 63 hogares; la mayoría de las unidades familiares se dedica principalmente al cultivo de la caña y diversas especies en huertos y, en menor escala, al cultivo de maíz, melón y sandía, además de que recolectan diversos productos en su entorno natural (Medina-Sánchez, 2000; Blanckaert, 2001; Rosas-López, 2003; Blanckaert et al., 2004; Lira et al., 2008).

Durante 2009, un total de 12 milpas de San Rafael fueron muestreadas con el método de línea de Canfield (Canfield, 1941; Blanckaert, 2007; Blanckaert et al., 2007). En cada milpa se evaluaron 10 líneas de $10 \mathrm{~m}$ de largo cada una y en cada línea se registraron todos los individuos de cada especie arvense que las interceptó. Para cada individuo detectado se midió la longitud de su sobreposición en la línea como una forma de estimar la cobertura (Bonham, 1989).

Las milpas que se muestrearon fueron las que estuvieron disponibles en el momento del estudio y, en consecuencia, no todas se encontraron en la misma etapa de desarrollo del cultivo (Cuadro 2). Todas las milpas son relativamente contiguas entre sí y están localizadas en sitios cercanos al poblado, y en todos los casos estaban sembradas únicamente con maíz; además, de acuerdo con la información proporcionada, todas ellas se irrigan aproximadamente cada 15 días mediante un sistema de riego por canales denominado apancle, el cual aprovecha agua del río Salado (Blanckaert, 2001; Rosas-López, 2003). Como también puede observarse en el Cuadro 2, con una sola excepción (milpa 5), los propietarios de las milpas indicaron no hacer uso de herbicidas para eliminar las plantas arvenses.

Con base en la información de los muestreos se obtuvo la riqueza (número de especies), abundancia (número de individuos de cada especie) y cobertura (centímetros de sobreposición de los individuos en la línea) para cada una de las milpas. También para cada milpa se calculó el índice de diversidad de Shannon, y la comparación de la riqueza entre las milpas se hizo mediante un análisis de conglomerados (Hoft et al., 1999).

Con el fin de evaluar si la riqueza y abundancia encontradas al momento de los muestreos estaban o no asociadas con el heterogéneo estado de desarrollo del cultivo en las milpas, se llevó a cabo un análisis de correlación de Spearman entre dicho factor y la riqueza y la abundancia de especies registradas en cada milpa. Por otra parte, debido a que la estimación de la diversidad depende significativamente del esfuerzo de muestreo, se elaboró una curva de acumulación de especies de todas las milpas para conocer la proporción de especies registradas de acuerdo con el esfuerzo de muestreo realizado (Colwell, 1997; Chazdon et al., 1998). Adicionalmente, se calculó la proporción de especies conocidas a partir de una riqueza total esperada, la cual se estimó iterando 50 veces la abundancia obtenida en cada milpa. Las iteraciones se hicieron utilizando el método Jackknife de primer orden por medio del programa EstimateS (Colwell, 1997).

Para determinar la dominancia de las especies en cada milpa, se hicieron curvas de rango abundancia, las cuales son un método para conocer la importancia relativa y la equidad de las especies, tomando en cuenta su identidad y abundancia relativa (Feinsinger, 2001). Para graficar las curvas se calculó el logaritmo natural de la proporción de la abundancia de cada especie y estos datos se ordenaron de mayor a menor (Feinsinger, 2001).

Los aspectos etnobotánicos se documentaron mediante entrevistas estructuradas a 20 agricultores, con edades entre 32 y 80 años. Estas personas son representantes del $30 \%$ de los 63 hogares de San Rafael y fueron elegidas siguiendo las recomendaciones de Tongco (2007), quien propuso que la selección de informantes para investigar un aspecto o dominio particular (en nuestro estudio el conocimiento de las plantas arvenses) se haga de acuerdo con la actividad que los califique como expertos de 
Cuadro 2. Ubicación geográfica y características generales de las 12 milpas muestreadas en San Rafael

\begin{tabular}{|c|c|c|c|}
\hline Milpa & Ubicación & Edad & Estado durante el muestreo \\
\hline 1 & $18^{\circ} 10^{\prime} 40.09^{\prime \prime} \mathrm{N} ; 9^{\circ} 09^{\prime} 03.54^{\prime \prime O}$ & 5 meses & Maíz en cosecha, $\mathrm{SH}$ \\
\hline 2 & $18^{\circ} 10^{\prime} 50^{\prime \prime} \mathrm{N} ; 9^{\circ} 08^{\prime} 23.4 ” \mathrm{O}$ & 1 mes & Maíz en inicio de desarrollo, SH \\
\hline 3 & $18^{\circ} 10^{\prime} 54^{\prime \prime N} ; 97^{\circ} 08^{\prime} 27.3 ” O$ & 3 meses & Maíz en desarrollo, SH \\
\hline 4 & $18^{\circ} 10^{\prime} 20.4^{\prime \prime} \mathrm{N} ; 9^{\circ} 08^{\prime} 35.4^{\prime \prime O}$ & 2 meses & Maíz en desarrollo, SH \\
\hline 5 & $18^{\circ} 10^{\prime} 57.9^{\prime \prime N} ; 97^{\circ} 08^{\prime} 26.3^{\prime \prime O}$ & 3 meses y 15 días & Maíz a punto de cosecharse, $\mathrm{CH}$ \\
\hline 6 & $18^{\circ} 11^{\prime} 08.7^{\prime \prime} \mathrm{N} ; 9^{\circ} 08^{\prime} 32.7^{\prime \prime} \mathrm{O}$ & 3 meses y 15 días & Maíz en desarrollo, $\mathrm{SH}$ \\
\hline 7 & $18^{\circ} 11^{\prime} 06.99^{\prime} \mathrm{N} ; 9^{\circ} 09^{\prime} 04^{\prime} \mathrm{O}$ & 3 meses & Maíz en desarrollo, SH \\
\hline 8 & $18^{\circ} 10^{\prime} 54.06^{\prime \prime} \mathrm{N} ; 97^{\circ} 08^{\prime} 50.3^{\prime \prime} \mathrm{O}$ & 3 meses y 15 días & Maíz en desarrollo, SH \\
\hline 9 & $18^{\circ} 10^{\prime} 37.5^{\prime \prime} \mathrm{N} ; 9^{\circ} 08^{\prime} 08.3 ” \mathrm{O}$ & 20 días & Maíz en inicio de desarrollo, $\mathrm{SH}$ \\
\hline 10 & $18^{\circ} 10^{\prime} 42.6^{\prime \prime} \mathrm{N} ; 9^{\circ} 08^{\prime} 20.9^{\prime \prime} \mathrm{O}$ & 2 meses y 15 días & Maíz en desarrollo, SH \\
\hline 11 & $18^{\circ} 10^{\prime} 56.6^{\prime \prime} \mathrm{N} ; 9^{\circ} 08^{\prime} 32.5^{\prime \prime} \mathrm{O}$ & 4 meses & Maíz a punto de cosecharse, $\mathrm{SH}$ \\
\hline 12 & $18^{\circ} 10^{\prime} 58.7^{\prime \prime} \mathrm{N} ; 9^{\circ} 08^{\prime} 29.3 ” \mathrm{O}$ & 3 meses & Maíz en desarrollo, SH \\
\hline
\end{tabular}

$\mathrm{SH}$, sin herbicida; $\mathrm{CH}$, con herbicida.

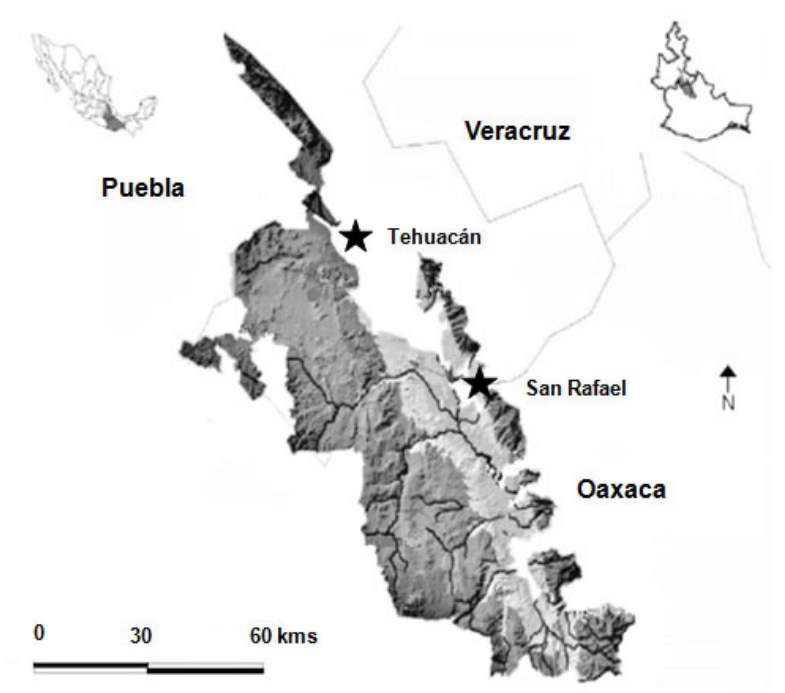

Figura 1. Ubicación de la comunidad de San Rafael, Municipio de Coxcatlán, Puebla.

dicho dominio. En las entrevistas se usaron como estímulo muestras herborizadas de las plantas encontradas en los muestreos. Para cada especie reconocida por los informantes se obtuvo información sobre los nombres comunes, usos y formas de manejo.

Algunos aspectos del conocimiento tradicional recopilado durante las entrevistas se evaluaron mediante métodos cuantitativos, siguiendo las recomendaciones de Begossi (1996), Lozada et al. (2006) y Hoffman y Gallagher (2007). Así, con el fin de evaluar si la muestra de personas entrevistadas era suficiente para documentar el conocimiento tradicional de la flora arvense encontrada en las milpas de San Rafael, se graficó una curva de acumulación de las especies reconocidas por los entrevistados y se calculó el número de especies esperado de acuerdo con el esfuerzo de muestreo realizado (número de informantes). También, en este caso, se utilizaron 50 iteraciones por medio del método Jacknife de primer orden con el programa EstimateS (Colwell, 1997). Finalmente, para poner a prueba la hipótesis referente a la relación entre la edad y el conocimiento tradicional, se realizó un análisis de correlación de Spearman entre la edad de los informantes entrevistados y el número de especies reconocidas por ellos durante las entrevistas.

De todas las plantas registradas en los muestreos se recolectaron ejemplares de herbario, los cuales serán depositados en el herbario IZTA. Vale la pena señalar que, dadas las condiciones heterogéneas de desarrollo de las milpas, algunos de los ejemplares recolectados no presentaron flores ni frutos (véase Cuadro 3). Los análisis multivariados y el cálculo de de los índices de diversidad se hicieron con la versión 2 del programa Biodiversity Pro (Mc Aleece, 1997), mientras que para los análisis de correlación se utilizó la versión 17 del programa SPSS (SPSS, 2008).

\section{Resultados}

Composición florística, riqueza y diversidad de las milpas. Se registró un total de 42 especies de plantas arvenses en las milpas muestreadas, de las cuales se pudo determinar que 40 pertenecen a 12 familias de plantas vasculares (Cuadro 3). De acuerdo con el análisis de acumulación de especies, esta riqueza representa el $70 \%$ del total de especies esperadas (60) en las milpas de San Rafael (Fig. 2).

Las familias con mayor número de especies fueron Poaceae (10 spp.), Asteraceae (7 spp.), Solanaceae (6 spp.) y Fabaceae (5 spp.), mientras que las especies mejor representadas en las milpas fueron Sonchus oleraceus (cerraja) y Portulaca oleracea (verdolaga), ambas presentes en 10 de las 12 milpas, así como Flaveria trinervia (hierba del sapo), encontrada en 9 de las milpas. Otras, como Setaria parviflora (cola de zorra) y Eragrostis pilosa (pasto chino), 


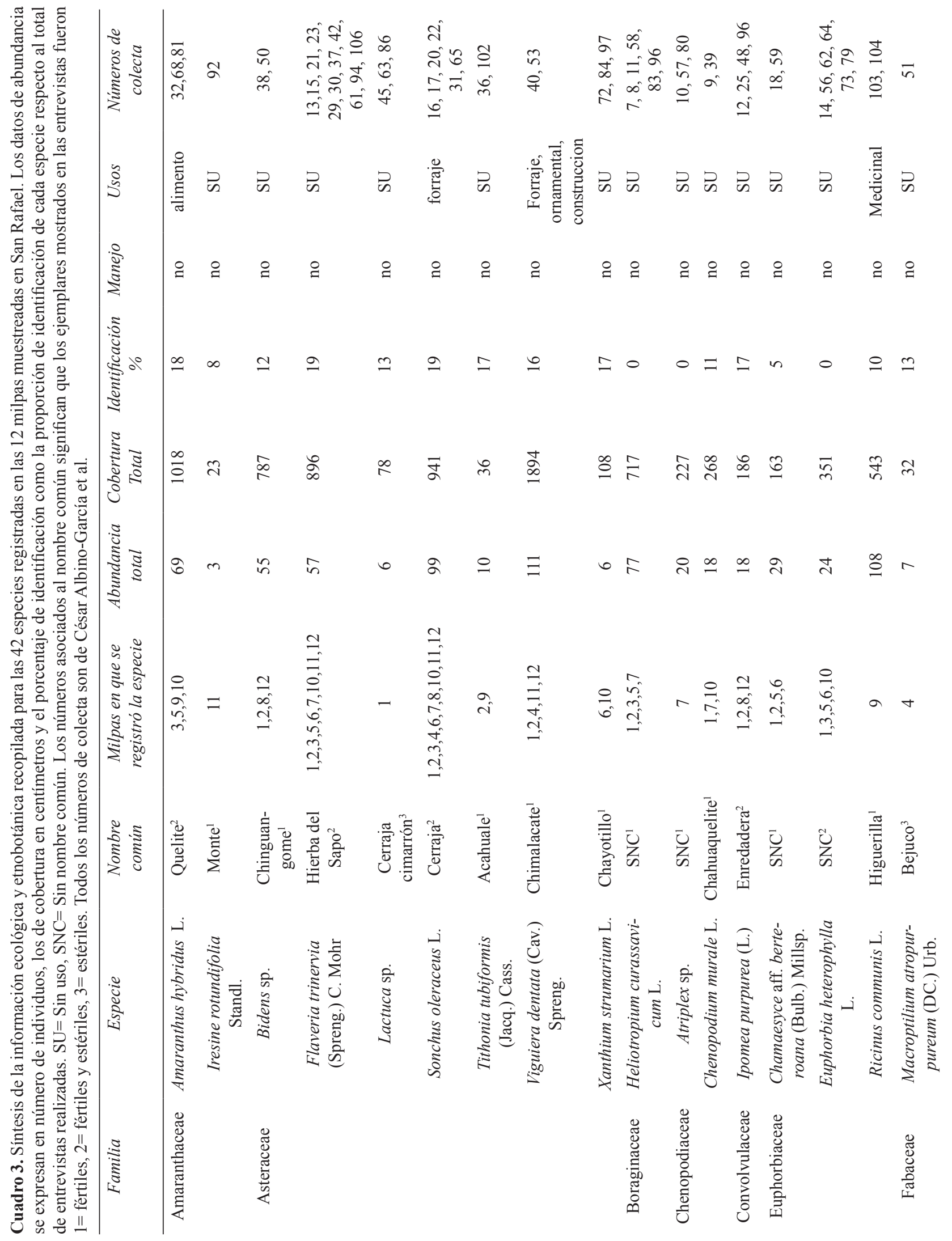




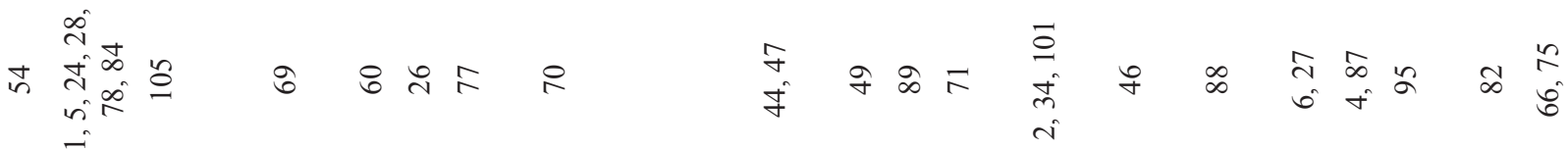

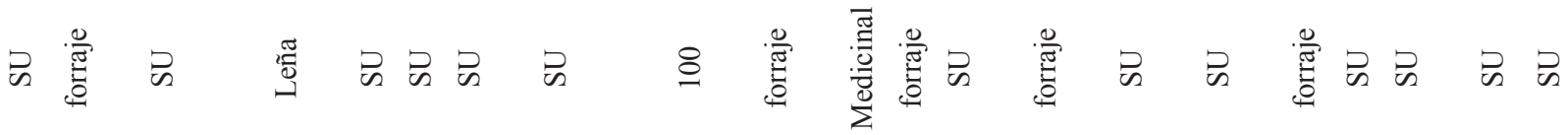

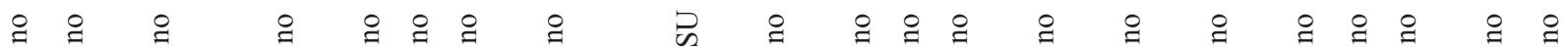

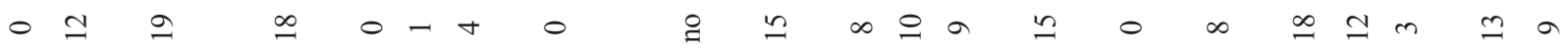

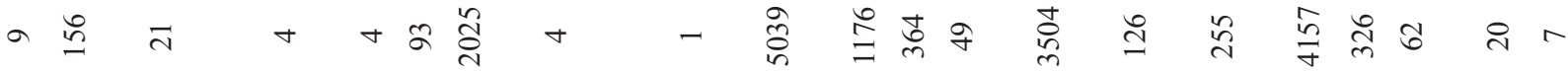

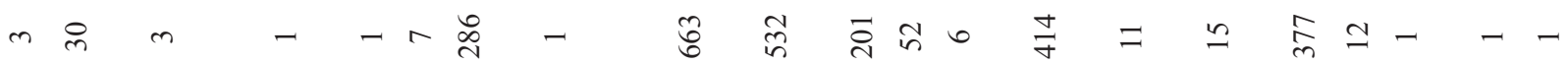

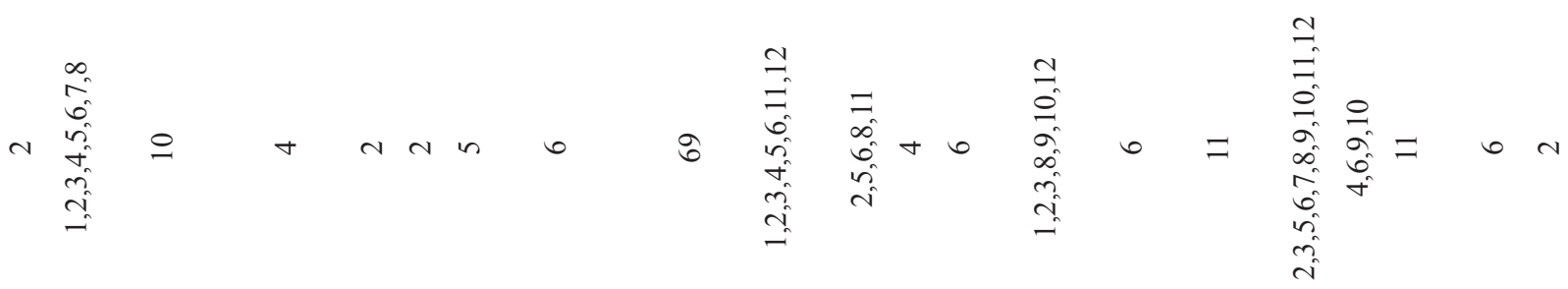

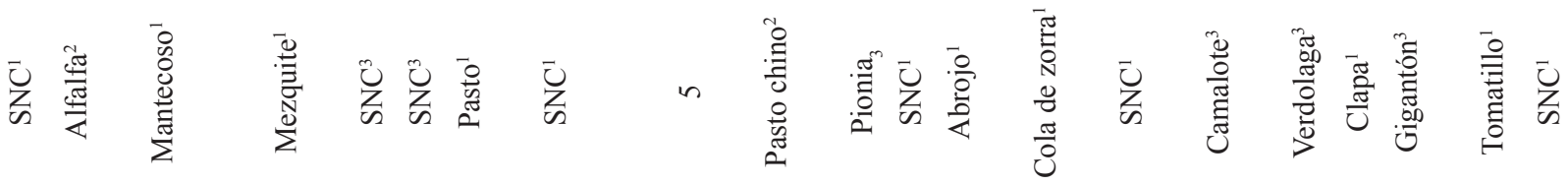

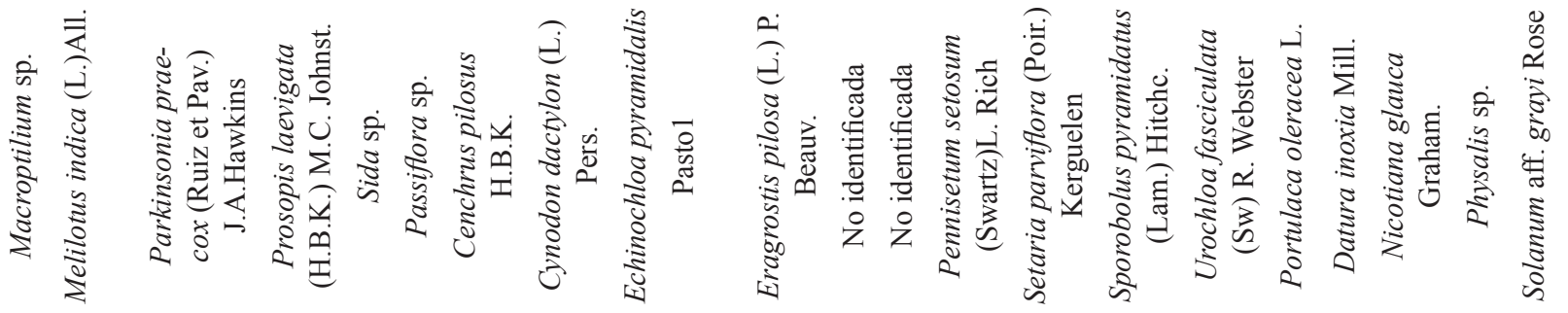

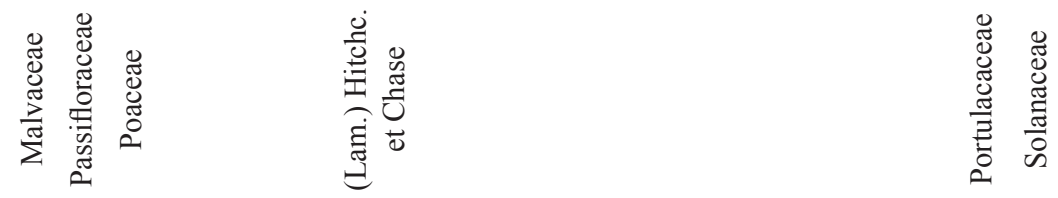




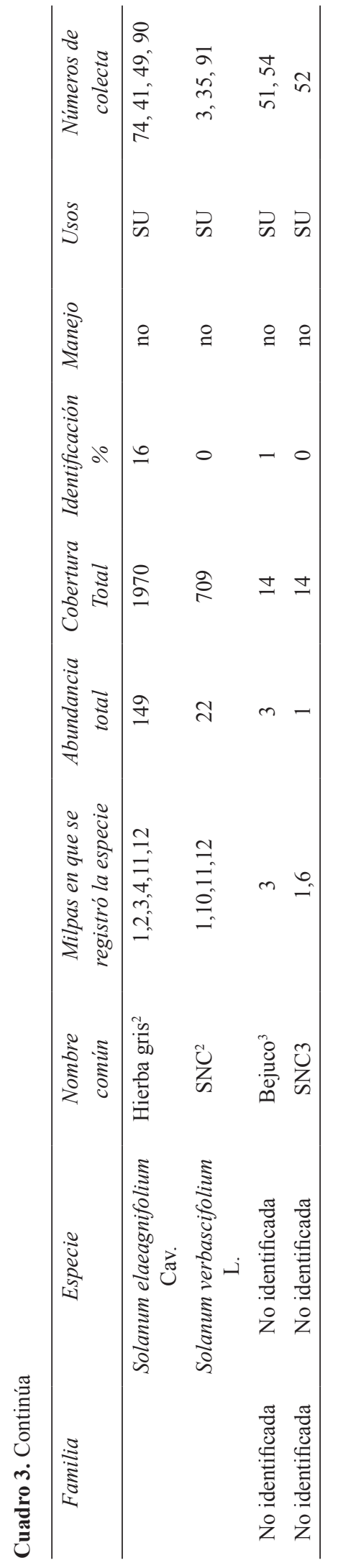

Cuadro 4. Valores de riqueza y diversidad calculados para las 12 milpas muestreadas en San Rafael. El índice de Shannon está calculado con el logaritmo natural

\begin{tabular}{ccccc}
\hline Milpa & Especies & Individuos & $\begin{array}{c}\text { Shannon H/ } \\
\text { Diversidad }\end{array}$ & $\begin{array}{c}\text { ShannonJ/ } \\
\text { Equidad }\end{array}$ \\
\hline M1 & 16 & 724 & 1.399 & 0.505 \\
M2 & 17 & 213 & 2.209 & 0.780 \\
M3 & 10 & 111 & 1.672 & 0.726 \\
M4 & 11 & 95 & 1.589 & 0.663 \\
M5 & 12 & 445 & 1.231 & 0.496 \\
M6 & 15 & 159 & 2.144 & 0.792 \\
M7 & 6 & 141 & 0.795 & 0.444 \\
M8 & 6 & 50 & 1.668 & 0.931 \\
M9 & 6 & 207 & 1.211 & 0.676 \\
M10 & 11 & 192 & 0.967 & 0.403 \\
M11 & 11 & 430 & 1.635 & 0.682 \\
M12 & 8 & 149 & 1.316 & 0.633 \\
x / 6 & $10.3 / 3.63$ & $243 / 194.18$ & $1.494 / 0.4440$ & $0.644 / 0.157$ \\
\hline
\end{tabular}

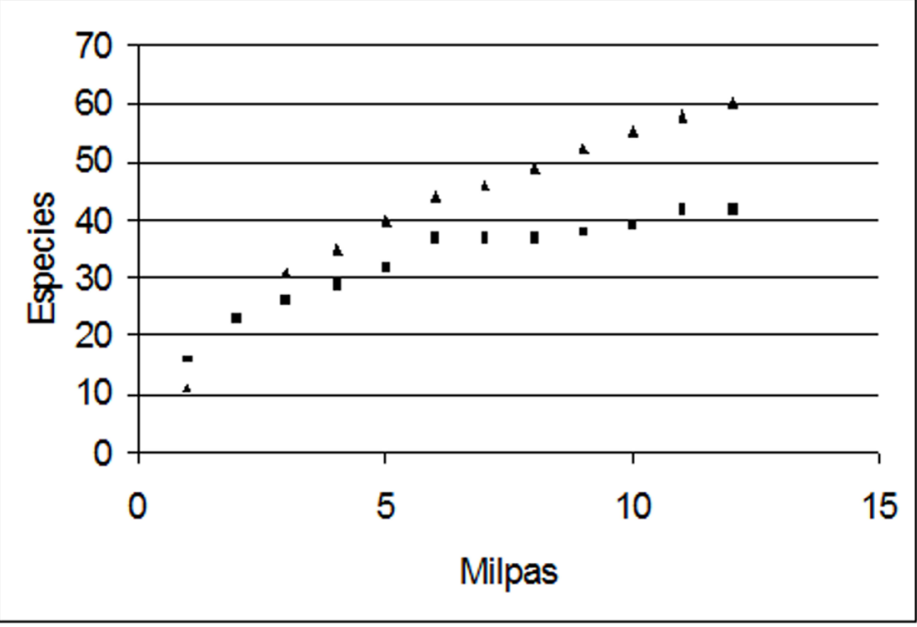

Figura 2. Curvas de acumulación de especies para las 12 milpas muestreadas; triángulos, esperada; cuadros, observada.

destacan por estar presentes en 7 y 8 de las milpas, respectivamente, así como por el alto número total de individuos (532 y 414, respectivamente) (Cuadro 3).

La distribución de las especies en las milpas fue heterogénea. En las milpas 7, 8, 9 y 12 se encontraron menos de 10 especies, mientras que en las 8 restantes se registraron entre 10 y 17 especies (Cuadro 4). Los valores de riqueza y diversidad de cada milpa también fueron variables, siendo las milpas 1, 2 y 6 en las que se encontró un mayor número de especies y en las 2 últimas donde se obtuvieron los valores más altos de diversidad (Cuadro 4). Las especies que presentaron los valores más altos de cobertura fueron 
Eragrostis pilosa, Portulaca oleracea, Setaria parviflora y Cenchrus pilosus.

De acuerdo con las curvas de rango-abundancia, se encontró que en todas las milpas unas cuantas especies fueron las más abundantes, mientras que la mayoría tuvieron valores de abundancia relativamente bajos (Fig. 3). Entre las especies dominantes, de acuerdo con su abundancia en las 12 milpas, destaca Portulaca oleracea en 3 de las milpas (6, 7 y 10), así como Sonchus oleraceus en 2 de las milpas (2 y 8), como se muestra en la figura 3 .

El análisis de conglomerados (Fig. 4) reveló que las milpas 1, 2, 3, 10, 11 y 12, son similares y forman un grupo relativamente bien definido, el cual está cercanamente asociado a otro grupo formado por las milpas 5 y 6 . Con mucho menor similitud está el grupo conformado por las milpas 7 y 8 , mientras que las milpas 4 y 9 son marcadamente disímiles a las de los grupos antes mencionados. Por otra parte, no se encontró relación entre la etapa de desa-

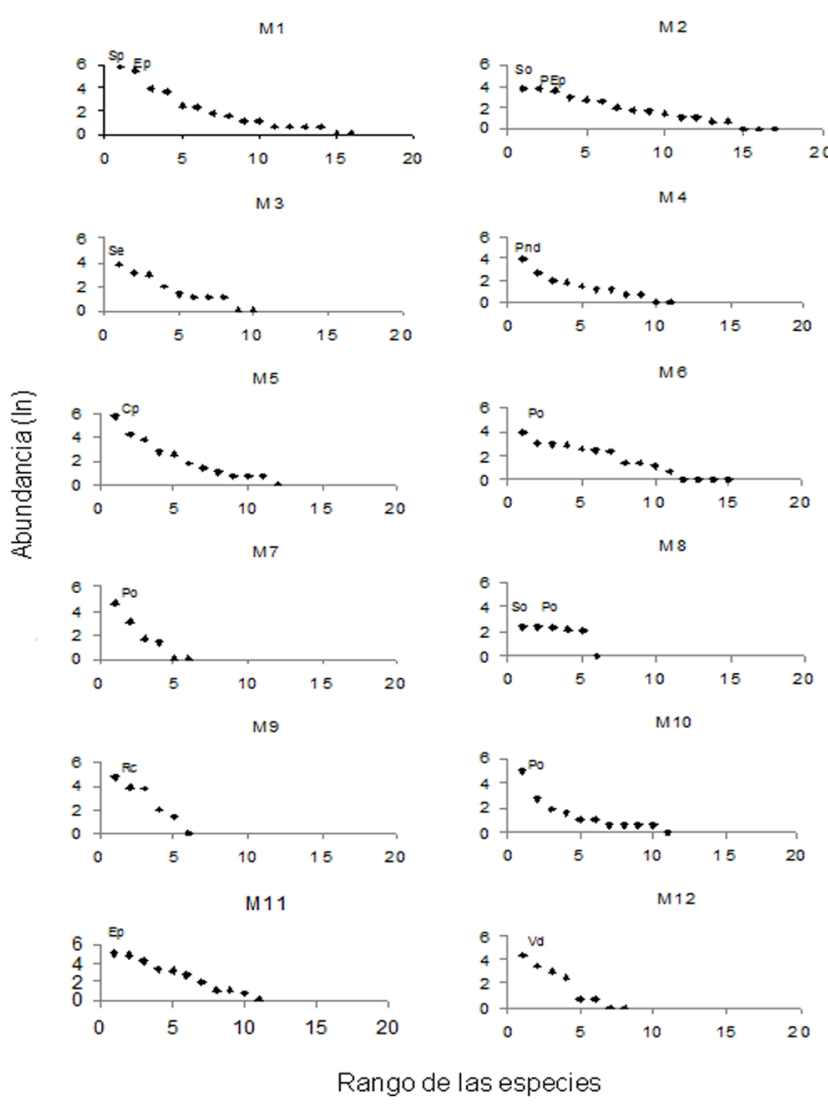

Figura 3. Curvas de rango-abundancia para las 12 milpas (M1 a 12) muestreadas en San Rafael. Para cada milpa se indica (n) la(s) especie(s) más abundante(s): Cp, Cenchrus pilosus; Ep, Eragrostis pilosa; P, pionia, Pnd, Poaceae no determinada; Po, Portulaca oleracea; Rc, Ricinus communis; Se, Solanum elaeagnifolium; So, Sonchus oleraceus; Sp, Setaria parviflora; Vd, Viguiera dentata. rrollo de las milpas y la riqueza y abundancia de especies de plantas arvenses $\left(\mathrm{r}_{\mathrm{s}}=0.17 \mathrm{n}=12 p=0.59 ; \mathrm{r}_{\mathrm{s}}=-0.21\right.$ $\mathrm{n}=12 p=0.51)$, ni con el índice de diversidad de Shannon $\left(\mathrm{r}_{\mathrm{s}}=-0.42 \mathrm{n}=12 p=0.89\right)$ o con la equitatividad $\left(\mathrm{r}_{\mathrm{s}}=-0.20\right.$ $\mathrm{n}=12 p=0.51$ ).

Conocimiento etnobotánico. Cada uno de los agricultores entrevistados reconoció entre 20 y 31 especies, aunque poco más de 14 fueron capaces de reconocer a 31 de las 42 especies registradas en los muestreos (Fig. 5). Considerando que el número de especies esperado con base en el esfuerzo de muestreo y las iteraciones realizadas fue de 36 , puede decirse que cerca de $86 \%$ de las especies esperadas fueron reconocidas por los agricultores entrevistados (Fig. 5). Por otra parte, no se encontró una correlación significativa entre el número de plantas identificadas y la edad de los informantes $\left(\mathrm{r}_{\mathrm{s}}=-0.071 \mathrm{n}=20 p=0.76\right)$ y el reconocimiento de las especies no dependió del estado de los ejemplares mostrados durante las entrevistas. Los informantes pudieron identificar algunas plantas aun cuando os ejemplares de herbario no presentaban flores y/o fruos, mientras que otras no fueron reconocidas ni siquiera 'n algunos de los casos en que los ejemplares estaban en :stado fértil (véase Cuadro 3).

La información obtenida en las entrevistas también eveló que la mayoría de los agricultores reconoció la nayor parte de las especies como malezas que afectan sus :ultivos. No obstante, 11 de las 42 especies fueron mencioradas como útiles, y algunas de ellas fueron reconocidas le esta forma por una alta proporción de los entrevistados. tsí, por ejemplo, 16 agricultores reconocieron a Sonchus leraceus, Portulaca oleracea, Eragrostis pilosa, Thitonia ubiformis y Viguiera dentata como plantas usadas para orraje; 18 mencionaron a Amaranthus hybridus como limento; 10 a Ricinus communis y la llamada pionia

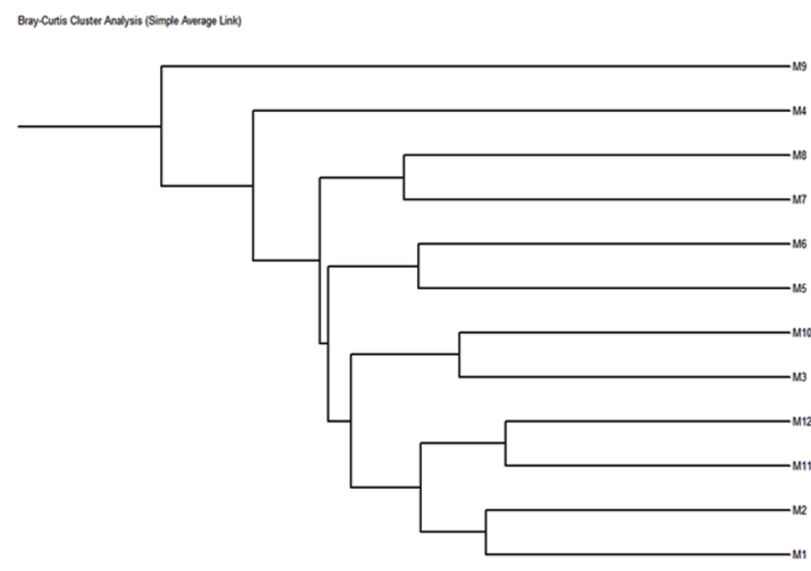

0. SS Simlaxicy 50.

Figura 4. Dendrograma de la similitud entre las milpas muestreadas en San Rafael. 


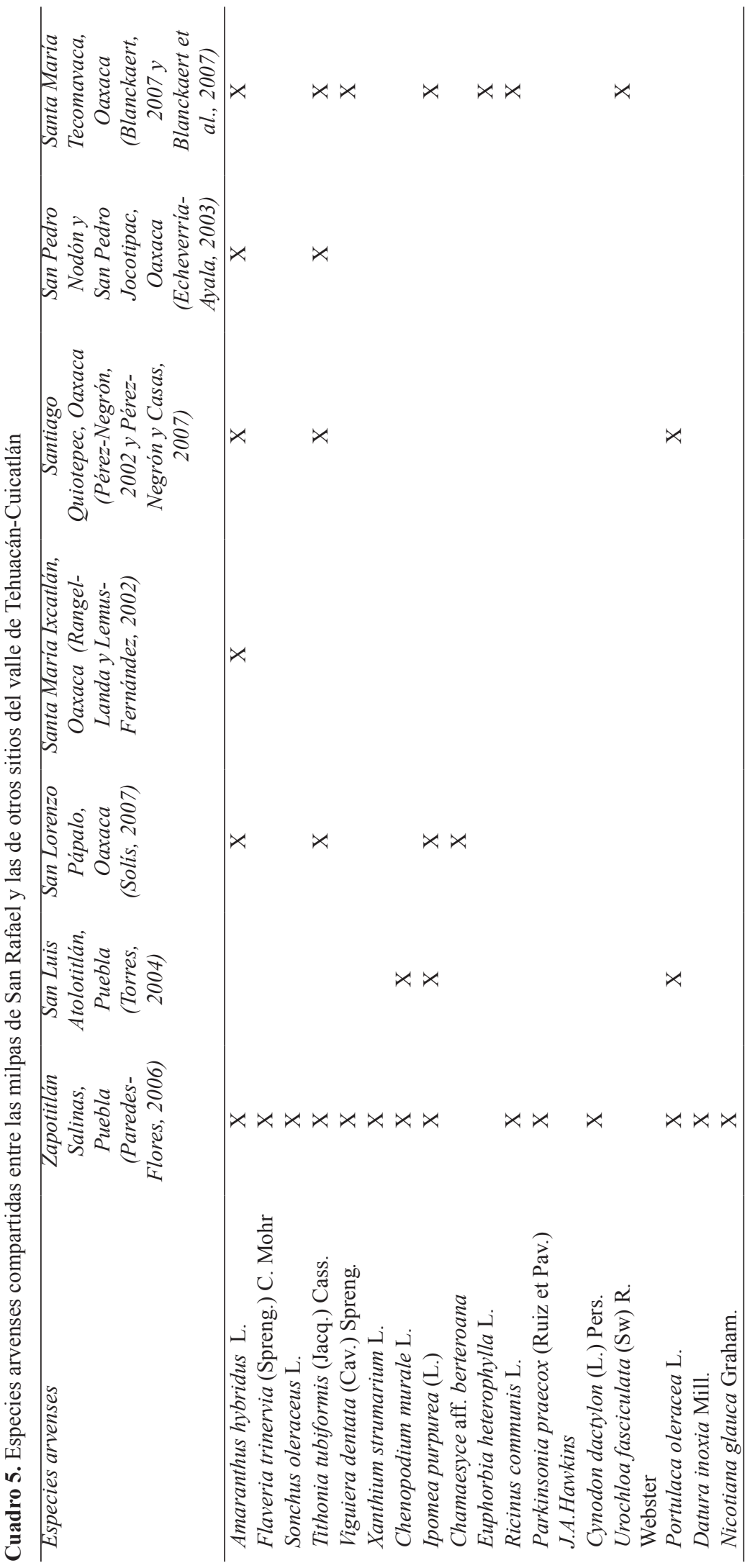

(Poaceae) como medicinales (para curar el empacho y la diabetes respectivamente) y 4 a Viguiera dentata como material de construcción.

Es importante destacar que, aun cuando no existe evidencia de manejo sobre ninguna de estas especies, todas ellas están entre las que tuvieron los mayores porcentajes de identificación por parte de los informantes entrevistados y, como ya se mencionó, algunas están presentes y son abundantes e incluso dominantes en varias de las milpas estudiadas (véanse Cuadro 3 y Fig. 3).

\section{Discusión}

Riqueza y diversidad de la flora arvense de San Rafael. La flora arvense documentada en las milpas de San Rafael presenta una composición relativamente esperada, pues $30 \mathrm{de}$ las 42 especies registradas en los muestreos (71\%) están incluidas en las listas de malezas registradas por Villaseñor y Espinosa (1998) para este sistema de cultivo en el estado de Puebla. Algunas especies registradas en los muestreos, como Prosopis laevigata, Datura inoxia y Nicotiana glauca, aunque estos autores las mencionan para el estado de Puebla, no las incluyen entre las listadas para campos de cultivo de maíz. Otras, como Ricinus communis, Mellilotus indica y Solanum eleagnifolium, los referidos autores las mencionan para cultivos de maíz pero no en el estado de Puebla. Y otras más, como Cenchrus pilosus, Echinochloa pyramidalis, Pennisetum setosum, Parkinsonia praecox, Solanum verbascifolium, Solanum aff. grayi y Chamaesyce aff. berteorana, no están registradas, ni para cultivos de maíz, ni para el estado de Puebla.

La riqueza de especies arvenses documentada en este trabajo se puede considerar intermedia respecto a la registrada en los estudios realizados en milpas de otras zonas del valle de Tehuacán (véase Cuadro 1). Así, como se muestra en el Cuadro 1, es mayor a lo registrado para 4 de los sitios, aunque considerablemente menor respecto a la que se ha registrado para Zapotitlán Salinas, Puebla, y Santa María Tecomavaca y San Lorenzo Pápalo en el estado de Oaxaca. Por otra parte, la comparación con esos trabajos 


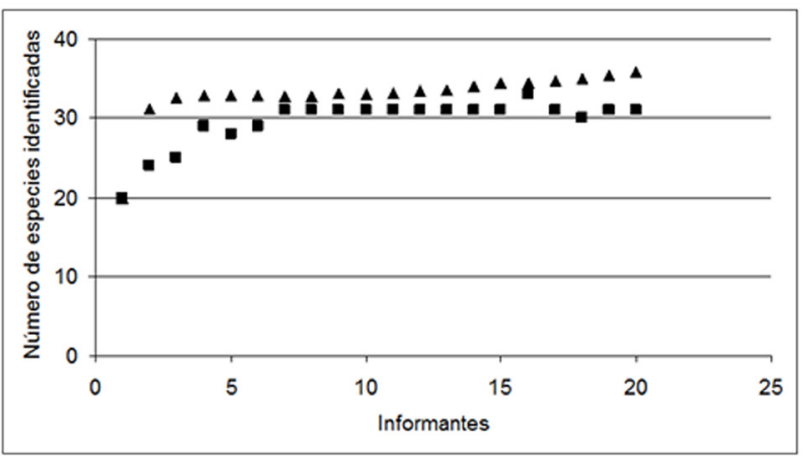

Figura 5. Curva de acumulacion de las especies de plantas arvenses identificadas por los 20 informantes entrevistados; triángulos, esperada; cuadros, observada.

en cuanto a la composición florística documentada en San Rafael, revela diferencias considerables, pues sólo unas cuantas especies son compartidas entre las milpas de los 7 sitios donde se realizaron esos estudios y las de San Rafael (Cuadro 5). De todas estas especies, destacan Amaranthus hybridus y Tithonia tubiformis, documentadas para la mayoría de los sitios, lo cual no resulta inesperado ya que se trata de 2 de las especies arvenses más comunes de los estados de Puebla y Tlaxcala (Vibrans, 1999).

En cuanto a la diversidad registrada mediante el índice de Shannon en las milpas de San Rafael, con sólo un par de excepciones (milpas 2 y 15, véase Cuadro 4), se puede considerar que está dentro de los límites esperados, ya que los valores calculados con el mismo índice para comunidades de arvenses en numerosos cultivos en general son menores de 2.0 (Légère et al., 2005). Desafortunadamente, no están disponibles los datos requeridos para hacer una comparación de este aspecto con los otros estudios realizados en el valle de Tehuacán-Cuicatlán.

Finalmente, la similitud entre la mayor parte de las milpas (Fig. 4), pudiera ser explicada por la proximidad entre ellas y, como sugiere (Vibrans, 1999), por la involuntaria dispersión por humanos, especialmente de algunas especies con diásporas con superficies que permiten su adherencia, o simplemente por las propias actividades de deshierbe y traslado hacia otros sitios de las plantas removidas. Para confirmar esta suposición, es necesario un análisis detallado de las características de las estructuras asociadas a la dispersión de las especies arvenses de San Rafael.

Las diferencias en métodos y/o intensidad del muestreo en los estudios con los que se ha hecho esta comparación pudieran explicar las notables diferencias en riqueza entre los resultados de esos trabajos y los encontrados en las milpas de San Rafael. Además, es pertinente mencionar que en cada uno de los sitios, factores asociados a las milpas, tales como su entorno climático y de vegetación, sus dimensiones, la disponibilidad de agua (por ejemplo, la precipitación pluvial o la intensidad y cantidad de riego), varios atributos del suelo y los tipos de cultivo que se manejan en ellas, así como su rotación, pueden ser determinantes en los patrones de riqueza de estas comunidades (Smith y Gross, 2007).

Aspectos etnobotánicos. La información etnobotánica documentada en este estudio también contrasta con la registrada en los trabajos similares efectuados en el valle de Tehuacán y otras regiones; por ejemplo, la ausencia de diferencias significativas entre el conocimiento de los informantes y su edad, difiere de lo que se informa en trabajos etnobotánicos de varios sitios del mundo, incluidos algunos de los realizados en la comunidad de San Rafael y otros sitios del valle de Tehuacán (Ladio y Lozada, 2001, 2003; Fassil, 2003; Canales et al., 2005, 2006; Carretero, 2005; Hernández et al., 2005; Blanckaert, 2007; Kamalakannan y Balakrishnan, 2009). Sin embargo, es acorde con lo encontrado por Arango-Caro (2004) para Colombia, Arias-Toledo et al. (2007) para Argentina, Stave et al. (2007) para Kenia y Lozada et al. (2006) para Patagonia. Una posible explicación a nuestros resultados es el hecho de haber elegido a los agricultores como informantes, los cuales se esperaba que tuvieran un conocimiento similar sobre las milpas. Además, como señala BlancoCastro (1996), no siempre las personas mayores tienen más conocimientos que los jóvenes, y las causas de estas diferencias en conocimiento merecen mayor investigación.

En cuanto al uso y manejo de las arvenses, las diferencias con los únicos estudios estrictamente comparables con este trabajo por los métodos utilizados (Blanckaert, 2007; Blanckaert et al., 2007), son también notables. Así, mientras en Santa María Tecomavaca se registraron 54 (98\%) de las 55 especies arvenses de las milpas con 1 o más usos, en San Rafael sólo 11 (26\%) de las 42 plantas arvenses fueron reconocidas como útiles. Adicionalmente, en contraste con las formas de manejo incipiente de distinta intensidad registradas para 7 las arvenses de Santa María Tecomavaca, ninguno de los entrevistados en San Rafael mencionó prácticas de manejo para mantenerlas (tolerancia, protección) o para incrementar (fomento o inducción) su abundancia en los campos de cultivo. No obstante estas importantes diferencias, existen coincidencias en cuanto al tipo de usos más importante de las plantas arvenses, pues al igual que en Santa María Tecomavaca, como ya se ha señalado, también la mayoría de las arvenses útiles de las milpas de San Rafael son destinadas al forraje (véanse Cuadros 1 y 3 ).

Aunque la información registrada en este trabajo no permitió corroborar la mayoría de las hipótesis planteadas, ofrece resultados importantes que sugieren que no es posible aún establecer generalizaciones en relación con 
los temas aquí estudiados para las plantas arvenses. Esto incluye las importantes diferencias entre lo registrado en San Rafael y otros estudios; por ejemplo, en los parámetros de riqueza de las milpas, los cuales pudiera suponerse que serían similares a los registrados en otros sitios, simplemente por estar regidos por las condiciones ambientales similares que les confiere un ambiente creado por el hombre como es la milpa. Como se señaló, seguramente otros factores asociados a las milpas e incluso a los métodos de obtención de la información, pudieran influir de manera significativa en dichos parámetros.

Tampoco debe generalizarse respecto al conocimiento tradicional referente al uso y, en especial, al manejo de estas plantas. Como han sugerido varios autores (Casas et al., 2001; Vieyra-Odilón y Vibrans, 2001; De Albuquerque y Lucena. 2005; González-Insuasti y Caballero, 2007; González-Insuasti et al., 2008; Lira et al., 2009), las decisiones acerca del uso y manejo de los recursos se toman con base en diversos factores biológicos, ecológicos y socioeconómicos, que aquí no se han tomado en cuenta, pero que seguramente varían entre culturas y aun dentro de una misma cultura.

Estudios más detallados en San Rafael, realizados en el marco de referencia de los trabajos antes mencionados, podrían, por ejemplo, contribuir a explicar por qué prácticamente todas las 11 especies arvenses útiles registradas en este trabajo, aunque no parecen estar sometidas a ninguna forma de manejo tradicional, no sólo estuvieron entre las que tuvieron los mayores porcentajes de identificación por parte de los informantes entrevistados, sino también entre las más frecuentes y abundantes en varias de las milpas estudiadas.

\section{Agradecimientos}

Este trabajo es parte del proyecto MGU - The Useful Plants Project - México, apoyado por Royal Botanical Gardens, Kew, a cargo de Rafael Lira -coautor- en la Unidad de Biotecnología y Prototipos de la Facultad de Estudios Superiores Iztacala, UNAM. Se agradece de manera muy especial a Ismael Calzada, a la Dra. Patricia Dávila Aranda y al Dr. Oswaldo Téllez Valdés (FES Iztacala, UNAM), por su generosa ayuda para la identificación de algunas especies de las familias Asteraceae, Leguminosae y Poaceae, así como al Dr. Alejandro Casas Fernández (Centro de Investigaciones en Ecosistemas, UNAM) y al Dra. Heike Vibrans (Colegio de Posgraduados en Ciencias Agrícolas), por sus muy valiosas recomendaciones para mejorar el trabajo.

\section{Literatura citada}

Alcorn, J. 1984. Huastec Mayan ethnobotany. University of Texas Press, Austin, Texas. 982 p.

Arango-Caro, S. 2004. Ethnobotanical studies in the Central Andes (Colombia): Knowledge distribution of plant use according to informant's characteristics. Lyonia 7:89-104.

Arias-Toledo, B., S. Colantonio y L. Galetto. 2007. Knowledge and use of edible and medicinal plants in two populations from the Chaco forest, Córdoba Province, Argentina. Journal of Ethnobiology 27:218-232.

Avendaño, A., A. Casas, P. Dávila y R. Lira. 2009. In situ management and patterns of morphological variation of Ceiba aesculifolia subsp. parvifolia (Bombacaceae) in the Tehuacán-Valley. Economic Botany 63:138-151.

Baker, H. G. 1965. Characteristics and modes of origin of weeds. In The genetics of colonizing species, H. G. Baker y G. L. Stebbins (eds.). Academic, New York. p. 147-172.

Begossi, A. 1996. Use of ecological methods in ethnobotany: Diversity indices. Economic Botany 50:280-289.

Blanckaert, I. 2001. An ethnobotanic survey of homegardens in San Rafael Coxcatlán, valley of Tehuacán-Cuicatlán, México. Master of science in tropical agriculture. Katholieke Universiteit, Leuven [Lovaina]. 83 p.

Blanckaert, I. 2007. Etnobotánica, ecología y posibles procesos de domesticación de malezas útiles en diferentes agroecosistemas en Santa María Tecomavaca, Oaxaca, México. Tesis doctorado, Posgrado en Ciencias Biológicas, Universidad Nacional Autónoma de México, México, D. F. $52 \mathrm{p}$.

Blanckaert, I., R. Swennen, M. Paredes, R. Rosas y R. Lira. 2004. Floristic composition, plant uses and management practices in homegardens of San Rafael Coxcatlán, valley of Tehuacán-Cuicatlán, Mexico. Journal of Arid Environments 57:179-202.

Blanckaert, I., K. Vancraeynest, R. Swennen, F. EspinosaGarcía, D. Piñero-Dalmau y R. Lira. 2007. Biodiversity of useful non-crop resources and the role of indigenous knowledge in their management in semi-arid crop production systems in Mexico. Agriculture, Ecosystems and Environment 119:39-48.

Blanco-Castro, E. 1996. Ideas metodológicas relativas al trabajo de campo etnobotánico. Monografías del Jardín Botánico de Córdoba 3:89-91.

Bonham, C. 1989. Measurements for terrestrial vegetation. Wiley, New York. 338 p.

Booth, B. D., S. D. Murphy y C. J. Swanton. 2003. Weed ecology in natural and agricultural systems. CABI, Cambridge, Massachusetts. 300 p.

Bye, R. 1979. Incipient domestication of mustards in northwest Mexico. The Kiva 44:237-256.

Bye, R. 1981. Quelites-ethnoecology of edible greens Past, 
present and future. Journal of Ethnobiology 1:109-123.

Bye, R. 1993. The role of humans in the diversification of plants. In Biological diversity in Mexico: origins and distribution, T. P. Ramamoorty, R. Bye, A. Lot y J. Fa (eds.). Oxford Univesity Press. New York. p. 707-731.

Bye, R. y E. Linares. 1983. The role of plants found in the Mexican markets and their importance in ethnobotanical studies. Journal of Ethnobiology 3:1-13.

Canales, M., T. Hernández, J. Caballero, A. Romo-De Vivar, G. Ávila, A. Durán y R. Lira. 2005. Informant consensus factor and antibacterial activity of the medicine plants used by the people of the San Rafael Coxcatlán, Puebla, México. Journal of Ethnopharmacology 97:429-439.

Canales, M., T. Hernández, J. Caballero, A. Romo-De Vivar, A. Durán y R. Lira. 2006. Análisis cuantitativo del conocimiento tradicional de las plantas medicinales en San Rafael, Coxcatlán, Valle de Tehuacán-Cuicatlán, Puebla, México. Acta Botanica Mexicana 75:21-43.

Canfield, R. H. 1941. Application of the line intercept method in sampling range vegetation. Journal of Forestry 39:388-394.

Cardina, J., G. A. Johnson y D. H. Sparrow. 1997. The nature and consequence of weed spatial distribution. Weed Science 45:364-373.

Carretero, A. L. 2005. Useful plants and traditional knowledge in the Tucumano-Boliviano forest. Tesis maestría, Institute of Biological Science University of Aarhus, [Dinamarca]. $56 \mathrm{p}$.

Casas, A., A. Valiente-Banuet, J. L. Viveros, J. Caballero, L. Cortés, P. Dávila, R. Lira e I. Rodríguez. 2001. Plant resources of the Tehuacán-Cuicatlán Valley, Mexico. Economic Botany 55:129-166.

Casas, A, J. Cruse, E. Morales, A. Otero-Arnaiz y A. ValienteBanuet. 2006. Maintenance of phenotypic and genotypic diversity of Stenocereus stellatus (Cactaceae) by indigenous people in Central Mexico. Biodiversity and Conservation 15:879-898.

Casas, A., A. Otero-Arnaiz, E. Pérez-Negrón y A. ValienteBanuet. 2007. In situ management and domestication of plants in Mesoamerica. Annals of Botany 100:1101-1115.

Colwell, R. K. 1997. EstimateS: Statistical estimation of species richness and shared species from samples, ver. 5, User's Guide and application. http://viceroy.eeb.unconn. edu/estimates; última consulta: 02.II.2010.

Chacón, J. C. y S. R. Gliessman. 1981. Use of the non-weed concept in traditional tropical agroecosystems of southeastern Mexico. Agro-Ecosystems 8:1-11.

Chazdon, R. L., R. K. Colwell, J. S. Denslow y M. R. Guariguata. 1998. Statistical methods for estimating species richness of woody regeneration in primary and secondary rain forest of NE Costa Rica. In Forest biodiversity research, monitoring and modeling: conceptual background and Old World case studies, F. Dallmeier y J. A. Comiskey (eds.). Parthenon,
Paris. p. 285-309.

Davis, T. y R. Bye. 1982. Ethnobotany and progressive domestication of Jaltomata (Solanaceae) in Mexico and Central America. Economic Botany 36:225-241.

De Albuquerque, U. P. y R. F. P. Lucena. 2005. Can apparency affect the use of plants by local people in tropical forests? Interciencia 30:506-511.

Dekker, J. 1997. Weed diversity and weed management. Weed Science 45:357-363.

Espinosa-García, F., J. L. Villaseñor y H. Vibrans. 2004. Geographical patterns in native and exotic weeds of Mexico. Weed Technology 18:552-1558.

Fassil, H. A. 2003. Qualitative understanding of local traditional knowledge and medicinal plant use (Ethiopia). Indigenous Knowledge Notes 52:1-4.

Feinsinger, P. 2001. Designing field studies for biodiversity conservation. The nature conservancy. Island, Washington, D. C. 212 p.

González-Insuasti, M. S. y J. Caballero. 2007. Managing plant resources: how intensive can it be? Human Ecology 35:303-314.

González-Insuasti, M. S., C. Martorell y J. Caballero. 2008. Factors that influence the intensity of non-agricultural management of plant resources. Agroforestry Systems 74:1-15.

Harlan, J. R., y J. M. J. De Wet. 1965. Some thoughts about weeds. Economic Botany 19:16-24.

Hernández, T., M. Canales, J. Caballero, A. Durán y R. Lira. 2005. Análisis cuantitativo del conocimiento tradicional sobre plantas utilizadas para el tratamiento de enfermedades gastrointestinales en Zapotitlán de las Salinas, Puebla, México. Interciencia 30:529-535.

Hoffman, B. y T. Gallagher. 2007. Importance indices in Ethnobotany. Ethnobotany Research \& Applications 5:201218.

Höft, M., S. K. Barik y A. M. Lykke. 1999. Quantitative ethnobotany. applications of multivariate and statistical analyses in ethnobotany. People and plants working paper. UNESCO, Paris, 49 p.

Kamalakannan, K. y V. Balakrishnan. 2009. Ethnobotanical studies on Achyranthes aspera Linn. among the folk peoples of Tamilnadu, South India. Journal of Phytology 1:108-111.

Ladio, A. H. y M. Lozada. 2001. Nontimber forest product use in two human populations from northwest Patagonia: a quantitative approach. Human Ecology 29:367-380.

Ladio, A. H. y M. Lozada. 2003. Comparison of wild edible plant diversity and foraging strategies in two aboriginal communities of northwestern Patagonia. Biodivesity and Conservation 12:937-951.

Légère, A., F. C. Stevenson y D. L. Benoit. 2005. Diversity and assembly of weed communities: contrasting responses across cropping systems. Weed Research 45:303-315. 
Liebman, M., C. L. Mohler y C. P. Staver. 2001. Ecological management of agricultural weeds. Cambridge University Press, Cambridge. 522 p.

Lira, R. y A. Casas. 1998. Uso y manejo en Ibervillea millspaughii (Cogn.) C. Jeffrey, Melothria pendula L. y otras especies silvestres de la familia Cucurbitaceae: posibles procesos de domesticación incipiente. Boletín de la Sociedad Botánica de México 62:77-89.

Lira, R., I. Rodríguez, L. García, H. Cervantes, C. M. Flores, J. Vázquez, I. Peñalosa, L. Hernández, M. Urzúa, M. Moreno, G. Avila, T. Hernández, M. Canales, A. M. García-Bores, R. Serrano, O. Coronado y M. López. 2008. 2o Informe del proyecto Conservación de Plantas Útiles de San Rafael Coxcatlán, a Través de Bancos de Semillas y Propagación, MGU/Useful Plants Project (UPP) - México, KEW Royal Botanic Gardens/FES Iztacala, UNAM, México. 20 p.

Lira, R., A. Casas, R. Rosas-López, M. Paredes-Flores, E. PérezNegrón, S. Rangel-Landa, L. Solís, I. Torres y P. Dávila. 2009. Traditional knowledge and useful plants richness in the Tehuacán-Cuicatlán Valley, México. Economic Botany 63:271-287.

Lozada, M., A. Ladio y M. Weigand. 2006. Cultural transmission of ethnobotanical knowledge in a rural community of Northwestern Patagonia, Argentina. Economic Botany 60:374-385.

Mapes C., F. Basurto y R. Bye. 1997. Ethnobotany of quintonil: knowledge, use and management of edible greens Amaranthus spp. (Amaranthaceae) in the Sierra Norte de Puebla, Mexico. Economic Botany 51:293-306.

Mc Aleece, N. 1997. Biodiversity Pro ver. 2. The Natural History Museum, London / The Scottish Association for Marine Sciences, Oban, Argyll.

Medina-Sánchez, J. 2000. Determinación del vigor y el estado reproductivo de Stenocereus stellatus (Cactaceae) a lo largo de una cronosecuencia edáfica en un abanico aluvial en Coxcatlán, valle de Tehuacán. Tesis, Facultad de Estudios Superiores-Iztacala, UNAM, Tlalnepantla, Estado de México. 48 p.

Paredes-Flores, M. 2001. Contribución al Estudio Etnobotánico de la Flora Útil de Zapotitlán de las Salinas, Puebla, México. Tesis, Facultad de Estudios Superiores Iztacala, UNAM, Tlalnepantla, Estado de México 104 p.

Paredes-Flores, M. 2006. Manejo, abundancia y variación morfológica del torito Proboscidea louisianica (Mill.) Thell. ssp. fragans (Lindl.) Bretting (Pedaliaceae), en Zapotitlán Salinas, Puebla. Tesis maestría, Posgrado en Ciencias Biológicas, Universidad Nacional Autónoma de México, México, D. F. 65 p.

Paredes-Flores, M., R. Lira y P. Dávila. 2007. Estudio etnobotánico de Zapotitlán Salinas, Puebla. Acta Botanica Mexicana 79:13-61.

Pérez-Negrón, E. 2002. Etnobotánica y aspectos ecológicos de las plantas útiles de Santiago Quiotepec, Cuicatlán, Oaxaca. Tesis, Facultad de Biología, Universidad Michoacana de San Nicolás de Hidalgo, Morelia, Michoacán. 284 p.

Pérez-Negrón, E. y A. Casas. 2007. Use, extraction rates and spatial availability of plant resources in the TehuacánCuicatlán Valley, Mexico: The case of Quiotepec, Oaxaca. Journal of Arid Environments 70:356-379.

Rangel-Landa, S. y R. Lemus-Fernández. 2002. Aspectos etnobotánicos y ecológicos de los recursos vegetales entre los Ixcatecos de Santa María Ixcatlán, Oaxaca. Tesis, Facultad de Biología, Universidad Michoacana de San Nicolás de Hidalgo, Morelia, Michoacán. 223 p.

Rodríguez-Arévalo, I., A. Casas, J. Campos y R. Lira. 2006. Uso, manejo y procesos de domesticación de Pachycereus hollianus (F.A.C. Weber) Buxb. (Cactaceae), en el valle de Tehuacán-Cuicatlán, México. Interciencia 31:677-685.

Rosas-López, R. 2003. Estudio etnobotánico de San RafaelCoxcatlán. Tesis, Facultad de Estudios Superiores Iztacala, UNAM, Tlalnepantla, Estado de México. 94 p.

Smith, R. G. y K. L. Gross. 2007. Assembly of weed communities along a crop diversity gradient. Journal of Applied Ecology 44:1046-1056.

Solís, L. 2006. Etnoecología cuicateca en San Lorenzo Pápalo, Oaxaca. Tesis maestría, Posgrado en Ciencias Biológicas, Universidad Nacional Autónoma de México, Mexico, D. F. $343 \mathrm{p}$.

SPSS (Statistical Package for the Social Sciences). 2008. Base 17.0 for Windows User's Guide. SPSS Inc., Chicago IL.

Stave, J., G. Oba, I. Nordal y N. Stenseth. 2007. Traditional ecological knowledge of a riverine forest in Turkana, Kenya: Implications for research and management. Biodiversity and Conservation 16:1471-1489.

Tongco, M. D. C. 2007. Purposive sampling as a tool for informant selection. Ethnobotany Research \& Applications 5:147-158.

Torres, I. 2004. Aspectos etnobotánicos y ecológicos de los recursos vegetales en la comunidad de San Luis Atolotitlán, municipio de Caltepec, Puebla, México. Tesis, Facultad de Biología, Universidad Michoacana de San Nicolas de Hidalgo, Morelia, Michoacán 198 p.

Vázquez-Rojas, M. C. 1991. Tendencias en el proceso de domesticación del papaloquelite (Porophyllum ruderale (Jacq.) Cass. subsp. macrocephalum (DC.) Johnson. Asteraceae). Tesis maestría, Facultad de Ciencias, Universidad Nacional Autónoma de México, México, D. F. $153 \mathrm{p}$.

Vibrans, H. 1999. Epianthropochory in Mexican weed communities. American Journal of Botany 86:476-481.

Vieyra-Odilón, L. y H. Vibrans 2001. Weeds as crops: the value of maize field weeds in the Valley of Toluca, México. Economic Botany 55:426-443.

Villaseñor, J. L. y F. J. Espinosa-García. 1998. Catálogo de 
malezas de México. Universidad Nacional Autónoma de México/ Consejo Nacional Consultivo Fitosanitario/ Fondo de Cultura Económica, México, D. F. 448 p.

Williams, D. E. 1985. Tres arvenses solanáceas comestibles y su proceso de domesticación en el estado de Tlaxcala, México.
Tesis maestría, Colegio de Postgraduados, Chapingo, Estado de México. 173 p.

Zizumbo-Villarreal, D. y P. Colunga-García-Marín. 1982. Aspectos etnobotánicos entre los Huaves de San Mateo del Mar, Oax. México. Biotica 7:223-270. 